\title{
VIBRATION ANALYSIS FOR VEHICLE WITH VACUUM PACKED PARTICLES SUSPENSION
}

\author{
Michae Makowski, Robert Zalewski \\ Warsaw University of Technology, Faculty of Automotive and Construction Machinery Engineering, Warsaw, Poland \\ e-mail:michal.makowski@simr.pw.edu.pl; robertzalewski@wp.pl
}

\begin{abstract}
The paper concerns an effect of the damping force controlling a vehicle suspension system. The investigated vehicle suspension system consists of innovatory Vacuum Packed Particles controllable dampers which allow controlling the vehicle body vibration. The main objective of performed and presented in the paper studies is to determine properties of the Vacuum Packed Particles (VPP) damper and propose a preliminary control strategy. The control in the vehicle suspension system can be realized by controlling the underpressure resulting in various jamming mechanisms in the granular core. Some algorithms of vibration control are also proposed and discussed.
\end{abstract}

Keywords: Vacuum Packed Particles, vibration damping, semi-active, smart materials

\section{Introduction}

For more than twenty years, innovative developments in the vehicle suspension design have focused on increasing the safety and comfort levels. For years, many automotive companies have carried out research aimed at continuous control of vehicles suspension. These efforts have resulted in a rapid development of advanced electronics and drive control systems as well as devices and structures which can be employed to achieve the goal.

At the moment, thanks to advances in measurement technology and microprocessor control, the development of a new generation of smart materials may lead to wide implementation of concepts of semi-active damping systems into everyday life. Especially interesting in this field seems to be application of technology based on the properties of the so called 'intelligent' materials including magnetorheological (MR) (Zalewski and Pyrz, 2013) electrorheological (ER) (Skup, 2009) and piezoelectric materials (Mikulowski et al., 2013). In this paper, the authors would like to focus on the new type of smart structures - Vacuum Packed Particles (VPP) (Zalewski and Pyrz, 2013). Former works of the authors aimed at the constitutive modelling of VPPs behaviour using both analytical (Zalewski, 2010) and stochastic identification procedures (Pyrz and Zalewski, 2010).

In the car industry, researchers and designers have long been investigating new ways to utilize the so-called "smart" suspension systems in order to reduce harmful vibrations. The essential part of existing solutions involved active or semi-active devices designed to dissipate kinetic energy in a controlled way. Smart suspension systems have suffered from the lack of a time-control loop putting accuracy of their control in question. It has been recently suggested that the time-control loop can be involved into intelligent suspension systems by means of semi-active magnetorheological dampers (MRDs) (Holnicki-Szulc et al., 2008; Makowski and Knap, 2013). Semi-active MRDs consist of magnetorheological fluids (MRF), which can significantly change their physical properties upon application of magnetic field. In late 1990s, Carlson and Goncalves (2008) introduced an MRD applicable to the on-and-off-highway vehicle suspension system. It was experimentally confirmed that the capability to control the damping force in a vehicle 
suspension system can be simply achieved by MRDs. Other researches proposed additional know-how in the field of developing effective vehicle and aircraft suspension systems with use of MRDs under different optimization criteria (Duysinx et al., 2005). At the same time, laboratory tests devoted to controlling the active suspension vehicle including various types of active or semi-active devices were carried out (March and Shim, 2007). It should be also mentioned that the controlled hydraulic dampers are now in-use in several manufactured vehicle suspension systems, where their dissipative features are adapted in time to the current vehicle load and road conditions (Prada et al., 2014).

In previous works by Bajkowski et al. (2012) main attention was paid to discussion of problems encountered during experimental research of MR dampers family. Conducting experiments on various MR devices, the authors found some similarities between MR fluids and vacuum packed particles (Zalewski and Pyrz, 2010).

The main aim of the present work is to investigate issues associated with control algorithms for an adaptive (semi-active) suspension system of a vehicle equipped with a new type of semi-active dampers. Numerical simulations results of the vehicle suspension system equipped with a VPP damper are presented and discussed in details.

\section{Experimental tests of granular cores}

According to previously mentioned similarities of MRFs and VPPs, the authors conducted a set of experiments on specially developed granular samples. The investigated granular cores are the main part of innovative granular damper prototypes used as a part of modelled vehicle suspension system. In the discussed experimental research of VPP, four different types of a loose material have been investigated (PMMA, polypropylene, polystyrene, ABS). The size and dimensions of a single grain are almost the same in each case. A typical grain is a cylinder having approximately $3 \mathrm{~mm}$ length and $1 \mathrm{~mm}$ in diameter.

The specially prepared experimental sample (Fig. 1) has been subjected to triangular displacement excitation (Fig. 5) and various underpressure values from 0.01 to $0.09 \mathrm{MPa}$.

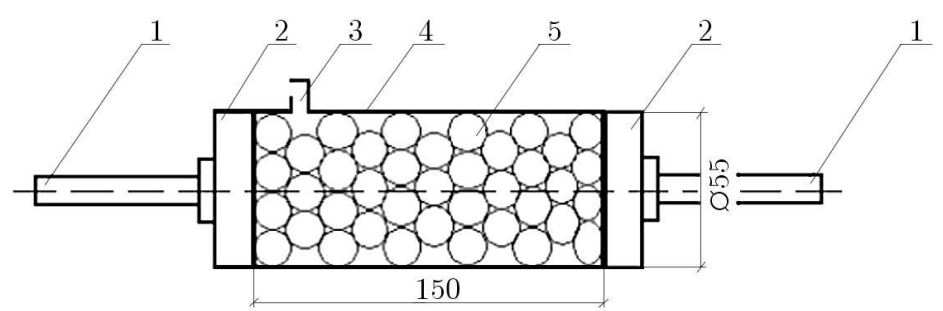

Fig. 1. Scheme of the special granular structure testing specimen; 1 - handle, 2 - steel disc, 3 - valve, 4 - encapsulation, 5 - grains

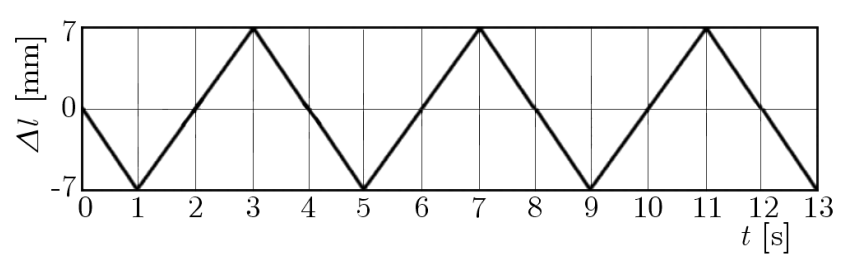

Fig. 2. Displacement-time triangular excitation

The excitation frequency has been $0.25 \mathrm{~Hz}$. Selected force responses for Polypropylene grains under a wide range of generated underpressures are shown in Fig. 3. All loading tests have been performed on the MTS 809 standard testing machine. 
It is worth mentioning that the applied in experimental research excitation program (Fig. 2) does not introduce inertial forces into the overall system except points, where the velocity changes direction. This allows for an accurate measurement of the force response.

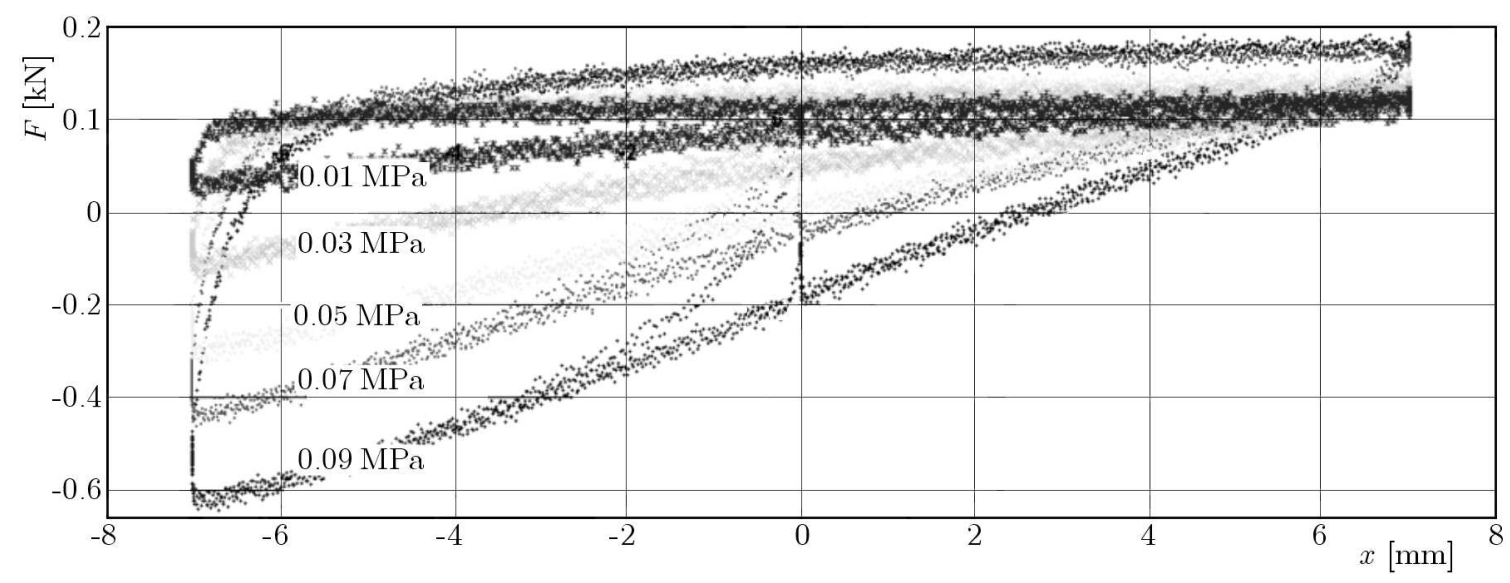

Fig. 3. Force-displacement diagrams for polypropylene grains and various underpressure values

An increase in the underpressure value results in an apparently larger force response. Measurements depicted in Fig. 3 reveal a significant difference in granular structures behaviour according to loading directions. Elongated granular structures behave totally different when compressed. This phenomenon is clearly reflected in a non-symmetrical shape of force-displacement diagrams illustrated in Fig. 3.

The force response for $0.09 \mathrm{MPa}$ is about 10 times greater (in the compression region) than for $0.01 \mathrm{MPa}$. The underpressure is a very convenient parameter for controlling the mechanical properties of vacuum packed particles.

\section{Granular damper design}

The main advantage of the considered granular damper prototype over popular controllable devices is the economical aspect. The granular material used in described prototypes is about 100 times cheaper in comparison to well commercialised MR shock absorbers.

Although granular devices could successfully compete with other popular solutions in selected engineering applications, during experimental research of granular systems a lot of additional problems can be encountered. Such problems are typical for innovatory or non-classical structures or engineering materials. Some of proposed solutions that enable their minimization or even negligence can be found in Zalewski and Pyrz (2013).

According to previously developed standards, a preliminary granular damper prototype has been designed. A basic scheme of its lower and upper bases also a global overview is depicted in Figs. 4 and 5, respectively.

\section{Model of the vehicle}

The mechanical model of a vehicle in form of a rigid plate is shown in Fig. 6. This simplified model is described by the oscillating mass $m$ and mass moments of inertia $I_{y}$, position of the center of mass $a_{1}$ and $a_{2}$. The parameter $x_{k}$ specifies the location of the checkpoint $K$ (for example, driver's position).

The investigated suspension system is also described by the spring stiffness $k_{1}$ and $k_{2}$, and shock absorbers with viscous friction characterized by damping coefficients $c_{T 1}, c_{C 1}$ and $c_{T 2}, c_{C 2}$. 

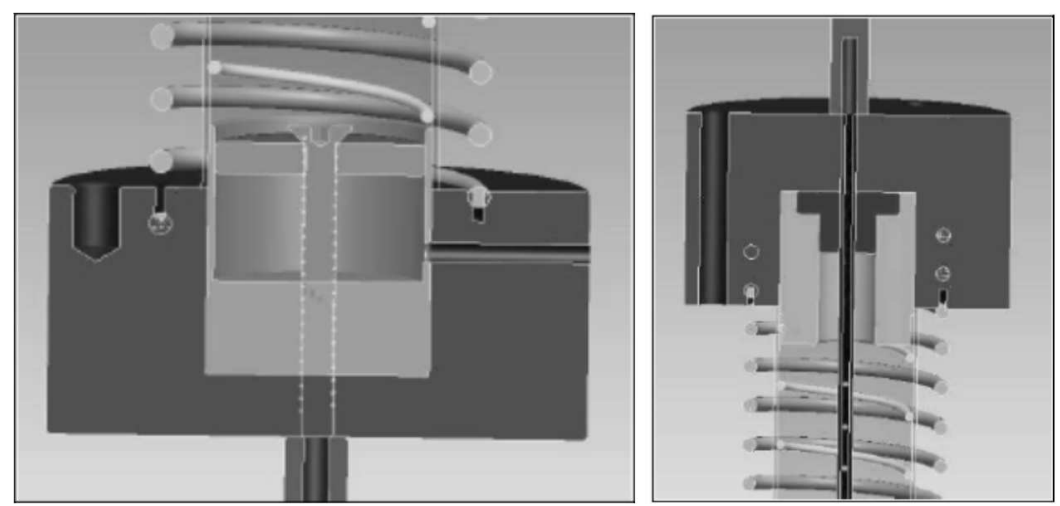

Fig. 4. Lower (left) and upper (right) bases of the investigated granular damper prototype

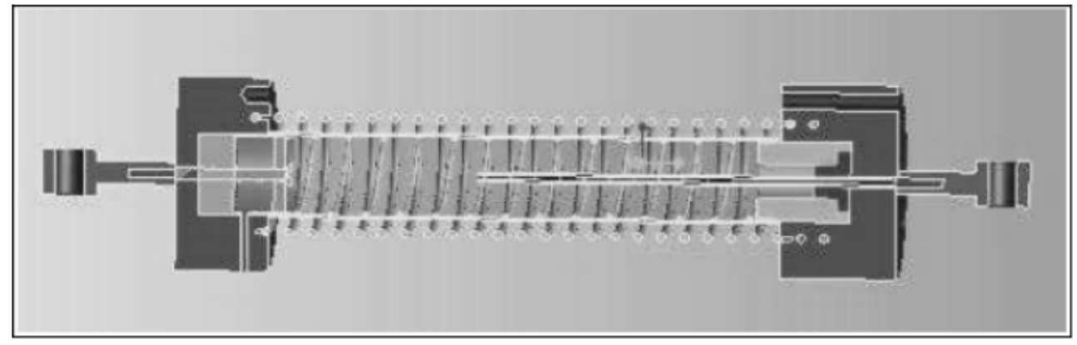

Fig. 5. Scheme of the granular damper prototype

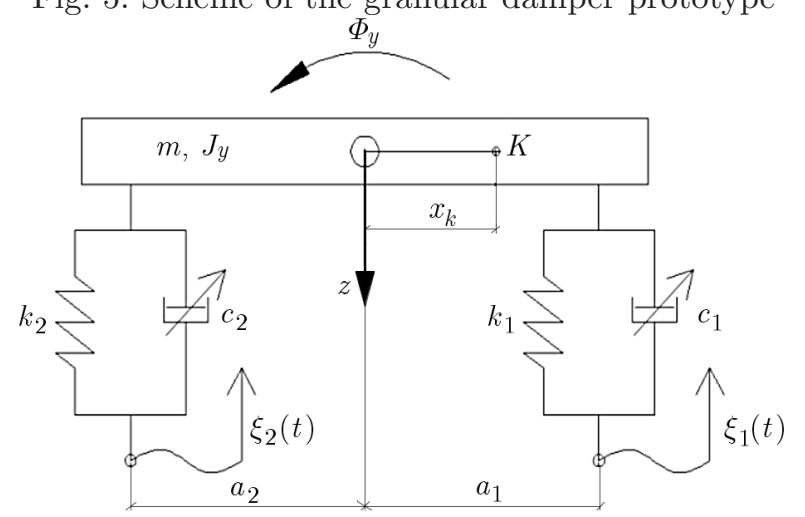

Fig. 6. Simplified model of a vehicle

The upper indexes $c$ and $t$ correspond to experimentally observed non-symmetrical damping characteristics of granular devices (Fig. 3). Vibrations of the vehicle model result from kinematic excitations described by functions $\xi_{1}(t)$ and $\xi_{2}(t)$.

Previous works of authors revealed the viscous-like behavior of Vacuum Packed Particles. For example, in Zalewski and Pyrz (2013) or Pyrz and Zalewski (2010) uniaxial tests conducted on granular cores were discussed. They showed that the response of the material is influenced by the strain rate applied in experiments. Additionally, in Zalewski and Szmidt (2014) the same phenomenon was found in periodically loaded samples. In the authors opinion the global viscous properties of VPP's are incorporated with nonlinear friction contacts occurring between single grains of the structure.

The vehicle model has been described in the coordinates

$$
\mathbf{X}=\left[z, \Phi_{y}\right]^{\mathrm{T}}
$$

where $X_{1}=z$ is the vertical displacement of vehicle, $X_{2}=\Phi_{y}$ - angle of rotation around the transverse axis of the vehicle. 
The flat model of the vehicle has been described by means of the following set of ordinary differential equations

$$
\mathbf{M X}+\mathbf{H}(\mathbf{S}+\mathbf{T})=\mathbf{0}
$$

where $\mathbf{M}=\operatorname{diag}\left(m, J_{y}\right)$. Matrix $\mathbf{H}$ describes the allowed configuration space to the forces $S_{i}$ and $T_{i}$

$$
\mathbf{H}=\left[\begin{array}{ll}
\mathbf{H}_{1} & \mathbf{H}_{2}
\end{array}\right]=\left[\begin{array}{cc}
1 & 1 \\
-a_{1} & a_{2}
\end{array}\right]
$$

The spring force $S$ and friction force $T$ in the damper are described by means of the following relationship

$$
\mathbf{S}=\left[S_{1}, S_{2}\right]^{\mathrm{T}}=\left[f_{1}\left(U_{1}\right), f_{2}\left(U_{2}\right)\right] \quad \mathbf{T}=\left[T_{1}, T_{2}\right]^{\mathrm{T}}=\left[F_{1}\left(V_{1}, w_{1}\right), F_{2}\left(V_{2}, w_{2}\right)\right]
$$

where $w_{i}(i=1,2)$ is the control parameter (the value of underpressure).

The values of suspension displacements $U_{i}$ and velocity $V_{i}(i=1,2)$ are calculated from following formulas

$$
U_{i}(t)=\mathbf{H}_{i}^{\mathrm{T}} \mathbf{X}+\xi_{i}(t) \quad V_{i}(t)=\mathbf{H}_{i}^{\mathrm{T}} \mathbf{X}+\dot{\xi}_{i}(t)
$$

where $\xi_{i}(i=1,2)$ are functions describing roughness of the pavement.

The damper forces $\mathbf{T}(t)$ are determined on the basis of the assumed control algorithm $F$, which is described later. Then, the equation of vibration of the vehicle turns into the following form

$$
\mathbf{M} \ddot{\mathbf{X}}+\mathbf{H S}(\mathbf{U})+\mathbf{H T}=\mathbf{0} \quad \mathbf{T}(t)=F(\mathbf{U}(t), \mathbf{V}(t))
$$

where $F$ is a function describing the algorithm for designating signals applied to the granular core.

Formula (4.9) describes the damper forces $\mathbf{T}(t)$ in the selected time moment. It is defined based on the proposed control strategy which is dependent on the control criteria presented in Section 5. The optimal friction force is determined on the basis of the deflection of the spring elements in the suspension and the current velocity value. Basing on the characteristic depicted in Fig. 7a for a selected value of the velocity, the $\mathbf{T}(t)$ force is chosen in such a way as to minimize the comfort indicator coefficient (5.5).

\section{Control algorithm}

A control algorithm has been developed assuming the need to meet two criteria: improvement of driving comfort and reduction of wheel force variations. The control of the comfort criterion can be achieved by minimizing the vertical body accelerations (which is a measure of exposure to fatigue resulted by vibration nuisance). However, there is a problem of criteria conflict. While the vehicle body acceleration is minimized, the force wheel variation grows significantly.

The control is possible in a given set of the allowed friction forces

$$
\begin{aligned}
& \mathbf{T} \in \Omega(V):=\left\{T_{i} \in \mathbb{R}^{1}, i=1,2\right\} \\
& T_{i}=\left\{\begin{array}{lll}
V_{i} c_{T i} & \text { for } \quad V \geqslant 0, & c_{T i} \in\left[c_{T \text { min }}, c_{T \text { max }}\right] \\
V_{i} c_{C i} & \text { for } \quad V<0, & c_{C i} \in\left[c_{C \text { min }}, c_{C \text { max }}\right]
\end{array}\right.
\end{aligned}
$$

Collection of the allowed friction forces $\Omega(V)$ is schematically shown in Fig. 7a. It is limited by a set of damping coefficients $c_{C \min }, c_{C \max }$ and $c_{T \text { min }}, c_{T \max }$ dependent on the speed $V$. 
The damping coefficients depend on the allowed underpressure values $p_{\min }$ and $p_{\max }$. In Fig. $7 \mathrm{~b}$, the real experimental data for the VPP's damper prototype is depicted. Various force-velocity characteristics have been recorded for different underpressure values. Additionally, the assumed values of $p_{\min }$ and $p_{\max }$ are illustrated. Basing on the experimental results, the idealized damping characteristic used in the numerical tests has been proposed (Fig. 7a).
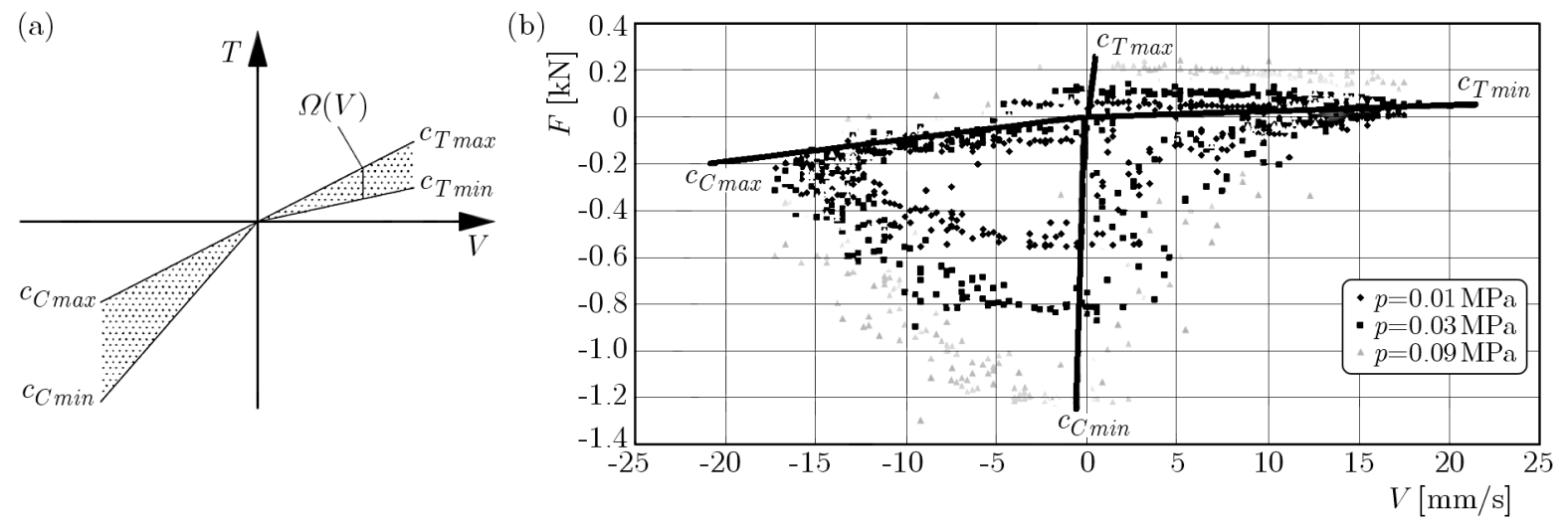

Fig. 7. (a) Set of the allowable solutions $\Omega(\mathbf{V})$, (b) real response of the granular damper

In the comfort criterion, the value of friction in any time is determined by minimizing an absolute value of the acceleration of the fixed point $K$, which corresponds (for example) to the position of the driver.

The value of this acceleration is described by the formula

$$
a_{k}=\mathbf{G}^{\mathrm{T}} \ddot{\mathbf{X}} \quad \mathbf{G}=\left[1, x_{k}\right]^{\mathrm{T}}
$$

where $x_{k}$ is the parameter specifies the location of the checkpoint $K, \mathbf{G}$ - vector determines the position of the point $K$.

On the basis of equation (4.2), the acceleration of the point $K$ is described by the following formula

$$
a_{k}=-\mathbf{G}^{\mathrm{T}} \mathbf{M}^{-1} \mathbf{H}(\mathbf{S}+\mathbf{T})=a_{0}(t)+\mathbf{D}^{\mathrm{T}} \mathbf{T}
$$

where

$$
\mathbf{D}^{\mathrm{T}}:=-\mathbf{G}^{\mathrm{T}} \mathbf{M}^{-1} \mathbf{H} \quad a_{0}(t):=\mathbf{D}^{\mathrm{T}} \mathbf{S}(\mathbf{U}(t))
$$

The comfort indicator is assumed and adopted in the form

$$
\kappa(\mathbf{T}, t)=\left|a_{0}(t)+\mathbf{D}^{\mathrm{T}} \mathbf{T}\right|
$$

The first criterion of minimizing the vehicle body acceleration is the optimization problem of finding the best friction force in the SGS damper. The problem is formulated in the following form

$$
T_{K}(t) \in \operatorname{Arg} \min \mathbf{T} \in \Omega(\mathbf{V})\left|a_{0}(t)+\mathbf{D}^{\mathrm{T}} \mathbf{T}\right|
$$

where Arg min is the set of solutions in which the minimized functional is convex but not strictly convex. A detailed description of the optimization problem solution and control algorithm is described in (Makowski and Knap, 2013). 


\section{Numerical simulation of the vehicle with SGS suspension}

In order to implement the optimal selection of friction forces in the granular damper, a simplified vehicle model has been developed in Matlab/Simulink environment.

The following values of parameters have been employed for the vehicle model in computer simulations: the oscillating weight $m=1200 \mathrm{~kg}$, spring stiffness $k_{1}=k_{2}=47500 \mathrm{~N} / \mathrm{m}$, $a_{1}=a_{2}=1.6 \mathrm{~m}$ and $x_{k}=0.5 \mathrm{~m}$. In order to solve the optimization problem, it has been necessary to define the limits of the dimensionless damping factor. According to the non-symmetrical characteristics of the VPP damper introducing the dimensionless damping coefficient $\gamma(6.1)$, the following parameters have been assumed in the controlling algorithm $\gamma_{C \text { min }}=0.05$ and $\gamma_{C \max }=1.0, c_{C \max }=750 \mathrm{Ns} / \mathrm{m}, c_{C \min }=15000 \mathrm{Ns} / \mathrm{m}, \gamma_{T \min }=0.017$ and $\gamma_{T \max }=0.3$ and $c_{T \min }=250 \mathrm{Ns} / \mathrm{m}, c_{T \max }=5000 \mathrm{Ns} / \mathrm{m}$. Additionally, numerical tests without control have been carried out for the constant $\gamma=0.3$ parameter $(c=4500 \mathrm{Ns} / \mathrm{m})$

$$
\gamma_{C}=\frac{c_{C i}}{2 \sqrt{k_{i} m}} \quad \gamma_{T}=\frac{c_{T i}}{2 \sqrt{k_{i} m}}
$$

The simulation studies have been carried out for a harmonic sinusoidal kinematic excitation defined by formula

$$
\xi_{i}=\xi_{0} \sin \left(\omega t+\beta_{i}\right) \quad i=1,2
$$

where

$$
\beta_{1}=0 \quad \beta_{2}=2 \pi\left(\frac{a_{1}}{a_{1}+a_{2}}\right)
$$

The numerical tests have been performed for the excitation frequency $2.25 \mathrm{~Hz}$ and amplitude $0.02 \mathrm{~m}$. The characteristic depicted in Fig. 8 presents acceleration of the point $K$ in versus time for the active and inactive controlling parameter.

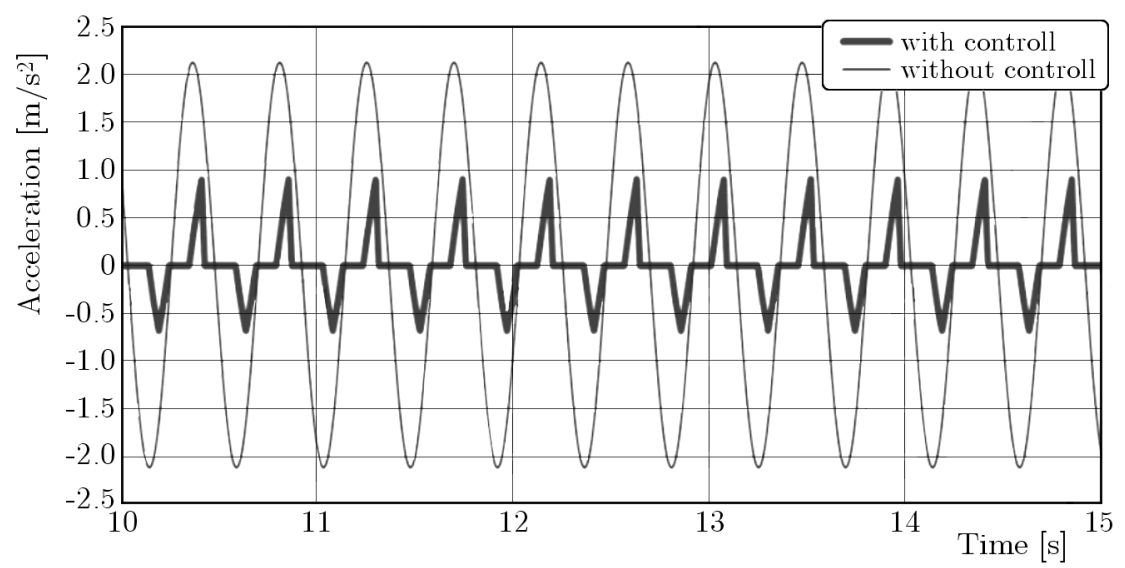

Fig. 8. Acceleration values of the selected $K$ point with and without application of the control algorithm $(f=2.25 \mathrm{~Hz})$

Basing on the above presented simulation results, lower acceleration values are observed for the controlled damping process according to the comfort criterion. In such a case, the damping coefficient is changeable in accordance to the applied excitation rule.

Numerical results for vertical accelerations of the selected point $K$ are depicted in Fig. 9. Similarly to the previous results, the controlling strategy provides much lower acceleration values in comparison to the non-controlled process. 


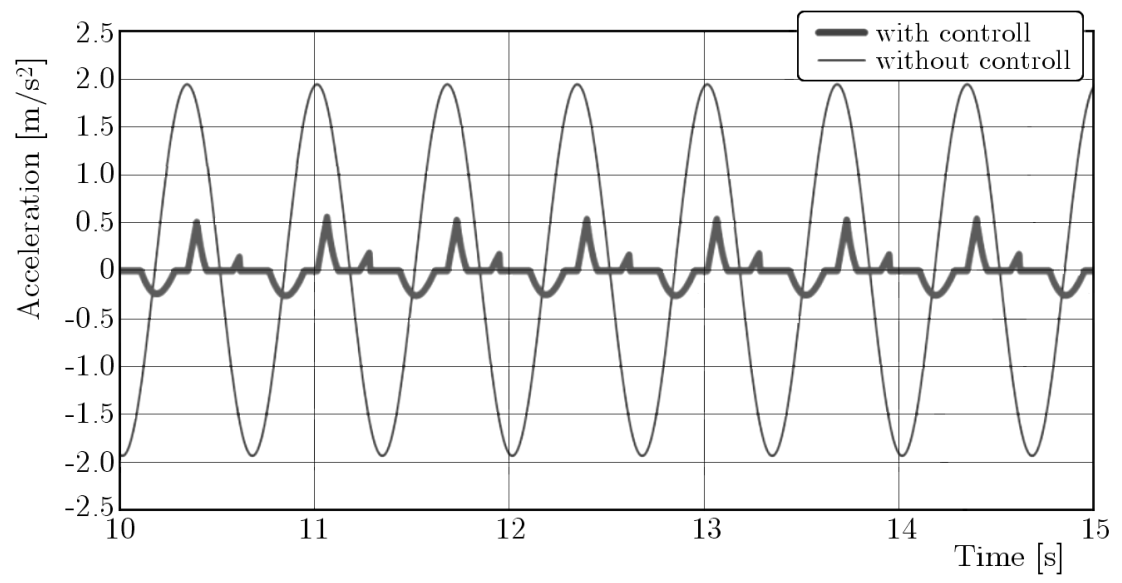

Fig. 9. Acceleration values for the selected $K$ point with and without application control algorithm $(f=1.5 \mathrm{~Hz})$

\section{Conclusions}

Results of numerical simulation and experimental investigations of a granular damper prototype have been presented in the paper. The experimental studies have been carried out to evaluate the granular damper dissipation characteristics.

The results of numerical simulations of the investigated devices have been used to propose a fast and efficient algorithm allowing improvements to passenger comfort. The proposed algorithm can be used to calculate the appropriate value of the signal to control semi-active devices with respect to two opposing criteria: comfort (reduction of vertical accelerations) and safety (reduction of wheel force variations).

The presented results of vehicle simulation demonstrated that it is possible to significantly reduce vertical accelerations using the controlled granular dampers (shock absorbers). The proposed vehicle model and control algorithm can be used to simulate other controlled dampers like magnetorheological, electrorheological or electrohydraulic. It is also possible to link the model and algorithm with external systems such as MBS Adams.

\section{References}

1. Bajkowski J., Jasiński M., Mączak J., RAdkowski S., Zalewski R., 2012, The active magnetorheological support as an element of damping of vibrations transferred from the ground to large-scale structure supports, Key Engineering Materials, 518, 350-357

2. Carlson J.D., Goncalves F., 2008, Controllable fluids come of age, 11th International Conference on New Actuators/5th International Exhibition on Smart Actuators and Drive, Conference Proceedings Book Series: Actuator-International Conference and Exhibition on New Actuators and Drive Systems, 477-480

3. Duysinx P., Bruls O., Collard J.F., Fisette P., Lauwerys J.S., 2005, Optimization of mechatronic systems: application to a modern car equipped with a semi-active suspension, 6th World Congresses of Structural and Multidisciplinary Optimization, Rio de Janeiro, Brazil

4. Holnicki-Szulc J., PawŁowski P., MikuŁowski G., Graczykowski C., 2008, Adaptive impact absorption and applications to landing devices, Advances in Science and Technology, 56, 609, 609-613

5. Makowski M., Knap L., 2014, Reduction of wheel force variations with magnetorheological devices, Journal of Vibration and Control, 20, 10, 1552-1564 
6. March C., Shim T., 2007, Integrated control of suspension and front steering to enhance vehicle handling, Proceedings of the Institution of Mechanical Engineers, Part D: Journal of Automobile Engineering, 221, 4, 377-391

7. Mikulowski G.,Wiszowaty R., Holnicki-Szulc J., 2013, Characterization of a piezoelectric valve for an adaptive pneumatic shock absorber, Smart Materials and Structures, 22, 125011 (12 pp)

8. Prada J.G., Vinolas J., CArrera X., 2014, Gas damper: potential vehicle performance studied on a full-car model, International Journal of Vehicle Design, 64, 214-239

9. Pyrz, M., Zalewski, R., 2010, Modeling of granular media submitted to internal underpressure, Mechanics Research Communications, 37, 2 141-144

10. Skup Z., 2009, Factors influencing load transmission through electrorheological cylinder clutch, Journal of Theoretical and Applied Mechanics, 47, 91-107

11. Zalewski R., 2010, Constitutive model for special granular structures, International Journal of Non-Linear Mechanics, 45, 3, 279-285

12. Zalewski R., Nachman J., Shillor M., Bajkowski J., 2014, Dynamic model for a magnetorheological damper, Applied Mathematical Modelling, 38, 2366-2376

13. Zalewski R., Pyrz M., 2010, Modeling and parameter identification of granular plastomer conglomerate submitted to internal underpressure, Engineering Structures, 32, 8, 2424-2431

14. Zalewski R., Pyrz M., 2013, Experimental study and modeling of polymer granular structures submitted to internal underpressure, Mechanics of Materials, 57, 75-85

15. Zalewski R., Szmidt T., 2014, Application of special granular structures for semi-active damping of lateral beam vibrations, Engineering Structures, 65, 13-20 